\title{
Phase II study of the antiretroviral activity and safety of the glucocorticoid receptor antagonist mifepristone in HIV-1-infected patients
}

\author{
MARCO DONIA $^{1}$, MASSIMILIANO ANZALDI ${ }^{1}$, ROBERTO DI MARCO ${ }^{2}$, MASSIMO LIBRA $^{1}$, \\ KATIA MANGANO $^{1}$, PAOLO FAGONE ${ }^{1}$, SALVATORE GALVAGNA ${ }^{3}$, \\ PIETRO DI GREGORIO ${ }^{3}$ and FERDINANDO NICOLETTI ${ }^{1}$ \\ ${ }^{1}$ Department of Biomedical Sciences, University of Catania, Catania; ${ }^{2}$ Department of Health Sciences, \\ University of Molise, Campobasso; ${ }^{3}$ Infectious Diseases Unit, Cannizzaro Hospital, Catania, Italy
}

Received February 24, 2011; Accepted April 7, 2011

DOI: $10.3892 /$ ijmm.2011.703

\begin{abstract}
Preclinical studies have shown that the antiglucocorticoid drug mifepristone effectively inhibits HIV replication both in vitro and in vivo. However, the drug did not demonstrate anti-HIV activity in a previous phase I/II study when administered at the daily dose of $75-225 \mathrm{mg}$. The aim of this study was to assess whether mifepristone may exert antiretroviral activity or influence immunological parameters when administered orally at daily doses of 150 or $300 \mathrm{mg}$ in highly active antiretroviral therapy (HAART)-naïve HIVinfected patients. We performed an open label non-randomized phase II study that included 26 patients who underwent 28 days of once daily oral administration of 150 (12 subjects) or $300 \mathrm{mg}$ (14 subjects) of mifepristone. A total of 3 patients dropped out of the study, respectively 1 in the $150 \mathrm{mg}$ dose group and 2 in the $300 \mathrm{mg}$ dose group. The main hematochemical alterations reported were hypokalemia and increase in the blood levels of cortisol, especially in those patients that received mifepristone at the dose of $300 \mathrm{mg} /$ day. Although we observed a trend of reduced viral load along the study in both groups, statistical significance was not achieved for either the primary nor the secondary endpoints. In summary, mifepristone treatment was well-tolerated but it failed to significantly influence viro-immunological parameters in HAART-naïve HIV-infected patients.
\end{abstract}

\section{Introduction}

One of the HIV-1 accessory proteins, the 96-aa virion associated accessory protein, viral protein $\mathrm{r}(\mathrm{Vpr})$, has been recently

Correspondence to: Professor Ferdinando Nicoletti, Department of Biomedical Sciences, Via Androne 81, I-95124 Catania, Italy

E-mail: ferdinic@unict.it

Key words: cortisol, HIV, glucocorticoid receptor, mifepristone, phase II, viral protein $\mathrm{r}$ implicated in a variety of functions. It has been demonstrated that $\mathrm{Vpr}$ is a vital constituent of the pre-integration complex (PIC), controls cellular functions, re-activates latent infection, and is involved in the infection of non-dividing target cells (1-5) (Muthumani K, et al, 12th CROI, 158, 2005).

Vpr enhances viral replication in monocyte- and lymphocytederived cell lines (6-8) and functions as a transcriptional activator of several viral promoters, including the HIV-1 long terminal repeat (LTR) promoter (9-12). Vpr is delivered to the infected cells with the viral particle at an early phase of HIV-1 infection, affecting infected lymphocytes, monocytes/macrophages, and dendritic cells (13). Thus, Vpr plays an important role in regulating the nuclear translocation of the HIV-1 preintegration complex, and it possibly helps HIV-1 to efficiently also infect nondividing cells, such as macrophages and resting lymphocytes (14-17). Moreover, it was recently shown that Vpr contributes to the innate and cellular immunity deficits of HIV-1-positive individuals and AIDS patients (17), since the polypeptide is also expressed and secreted by infected cells of the host after successful integration of the HIV-1 provirus into the host genome (18).

It is thought that most $\mathrm{Vpr}$ functions are due to the interaction with the cellular glucocorticoid receptor (GR) (4) Vpr enhances GR2 activity functioning as a potent coactivator of the GR, through a classic LXXLL coactivator signature motif $(10,17)$.

It was previously reported that the GR2 antagonist, mifepristone (or RU486), that is currently used as an abortive drug, may inhibit Vpr-induced transactivation of the HIV-1 LTR in a dose-dependent manner (4). Infectivity assays using X4 and R5 viruses demonstrated the dose-dependent antiviral effects of mifepristone regimen. Mifepristone abolished (>95\%) viral replication in primary human peripheral blood mononuclear cells (PBMC)s at a concentration of $10 \mu \mathrm{M}$, whereas a $60-70 \%$ inhibitory effect was observed at a concentration of $1 \mu \mathrm{M}$ (4). The effects of mifepristone were also examined in latently infected cells that could be activated with extracellular Vpr protein and the results indicated a specific inhibition of virus reactivation in the presence of this antagonist (4). Furthermore, in a simian immunodeficiency virus (SIV)-infected 
macaque model mifepristone injected intravenously clearly exhibited an antiviral activity (19).

Mifepristone is an orally available small molecule drug. Pre-existing toxicity data in animals have shown mifepristone to be safe at very high doses $(5 \mathrm{mg} / \mathrm{kg}$ for 6 months in rats and macaques). In addition, chronic administration of up to $200 \mathrm{mg} /$ day mifepristone for the experimental treatment of a variety of malignant and non-malignant conditions has been well tolerated in non-HIV-infected subjects for up to 1 year (20-24).

A previous phase I/II trial performed to assess the safety and anti-HIV activity of mifepristone concluded that a $75-225 \mathrm{mg}$ mifepristone daily dose over a 28 -day period was safe and well-tolerated, but failed to significantly influence plasma HIV-1 RNA levels and to alter CD4+ lymphocyte count from baseline levels (25).

To rule out the possibility that the lack of effect was due to the selection of an inappropriate dose regimen, we administered mifepristone to HIV patients at the two different doses of 150 and $300 \mathrm{mg}$ day for 4 consecutive weeks. Our data show that similarly to the previous trial, this different dosing of mifepristone did not exhibit antiretroviral activity (decrease in plasma HIV-primary endpoint) and did not influence $\mathrm{CD}^{+}$and $\mathrm{CD} 8^{+}$ T-cell counts (secondary endpoint) in HIV patients.

\section{Materials and methods}

Patients. To assess the antiretroviral efficacy of mifepristone in HIV-infected patients we performed an open label study including 26 patients that underwent 28 days of once daily oral administration of 150 (12 subjects) or $300 \mathrm{mg}$ (14 subjects) of mifepristone.

Protocol inclusion criteria were: age $\geq 18$ years; HIV-1 infection documented by ELISA test and confirmed by Western blot analysis at any time prior to study entry (HIV-1 culture, HIV-1 antigen, plasma HIV-1 RNA or a second antibody test by a method other than ELISA was acceptable as an alternative confirmatory test); $C D 4^{+}$cell count $\geq 200 / \mathrm{mm}^{3}$ obtained within 90 days prior to study entry; plasma HIV-1 RNA of $\geq 2000$ copies/ml as measured by any standard assay and performed within 90 days prior to study entry; stable hepatic (AST, ALT and alkaline phosphatase $\leq 2 \mathrm{x}$ ULN), renal (creatinine $\leq 2 \mathrm{x}$ ULN), and hematological (hemoglobin $\geq 10.0 \mathrm{~g} / \mathrm{dl}$ ) indices obtained within 30 days prior to study entry; negative spot urine pregnancy test result (girls who have reached menarche or women who have not been postmenopausal for at least 24 consecutive months); use of two reliable methods of contraception simultaneously while on study drug and for 30 days after stopping the medication; and a Karnofsky performance score $\geq 80$ within 30 days prior to study entry. Furthermore, any precautionary concomitant medications were to be on stable doses for $>8$ weeks prior to study entry with no plans to change medications or doses for the entire duration of the study.

Main exclusion criteria were the following: receipt of antiretroviral treatment within the 4 weeks prior to study entry or intent to initiate antiretroviral therapy within 60 days after entry; chronic adrenal failure; history of active hepatitis $\mathrm{B}$ or $\mathrm{C}$; porphyrias; known moderate to severe cirrhosis; hemorrhagic disorders; concurrent anticoagulant therapy; any prior pituitary tumor, surgery, radiation treatment or pituitary failure; diabetes requiring treatment with oral hypoglycemics or insulin therapy; pregnancy within 90 days prior to study entry; breastfeeding and dysfunctional uterine bleeding within the 12 months prior to study entry. Subjects who were using other investigational agents, vaccines, chemotherapies, some antiarrhythmics, anticonvulsants, hormonal agents (systemic corticosteroids, estrogens and progesterones) and/or cytochrome P450 3A4 (CYP3A4) inhibitors or inducers were also excluded.

Trial design. Patients were screened -90 to -14 days prior to receiving drug and monitored on a weekly basis for safety and antiviral effects. The study was conducted at the Infectious Disease Unit, Cannizzaro Hospital, Catania, Italy and was approved by the local ethics committee. Patients were divided into two treatment doses groups: the $150 \mathrm{mg}$ mifepristone once daily (75 mg, 2 tablets) and the $300 \mathrm{mg}$ once daily (75 mg, 4 tablets). Mifepristone was supplied from Viral Genomix Pharmaceuticals, Inc. (Philadelphia, PA, USA). Patients were visited at the clinic at weekly intervals (Days 0, 7, 14, 21, 28 and, then Day 56) for evaluation of safety and to obtain a peripheral blood sample to assess viral loads and $\mathrm{CD} 4^{+} / \mathrm{CD}^{+}$ T-cell counts. A general clinical visit and blood sampling for determination of routine hematochemical analyses were also performed. The latter included routine hematology, glucose, creatinine, total proteins, bilirubin, $\mathrm{Na}^{+}, \mathrm{K}^{+}$, transaminases, $\mathrm{LDH}$, amylases and cortisol determination. Tablet counts were performed at all study visits after the study medication was dispensed until subjects either completed or discontinued study treatment.

The following conditions/events were considered to result in treatment discontinuation: grade $\geq 3$ drug-related toxicity, development of a malignancy requiring systemic chemotherapy, pregnancy, requirement for prohibited concomitant medications, failure by the subject to attend two consecutive clinic visits, $\geq 3$ missed doses of study medication and clinical reasons believed to be life threatening by the physician, even if not addressed in the toxicity management of the protocol.

Statistical analysis. The primary endpoint of the study was the evaluation of the antiretroviral activity of mifepristone, defined by the change in HIV-1 plasma viral load between baseline and Days 7, 14, 21, 28 and 56. The secondary endpoints were the effects of mifepristone on the immunological parameters measured as changes in the absolute numbers and percentages of $\mathrm{CD}^{+}$and $\mathrm{CD}^{+} \mathrm{T}$ lymphocytes relative to baseline levels. Data are expressed as mean \pm SD. Statistical analysis was carried out by one-way ANOVA with Bonferroni's adjustement. P-values $<0.05$ were considered statistically significant.

\section{Results}

After the enrollment phase, the resulting 26 patients were randomly divided into 2 treatment arms (12 in the $150 \mathrm{mg}$ dose group and 14 in the $300 \mathrm{mg}$ dose one). A total of 23 patients completed the study follow-up. The main clinical and laboratory characteristics of these patients are summarized in Table I.

In the $150 \mathrm{mg}$ dose group, there was only one drop-out patient who discontinued treatment on Day 7 because of elevated 
Table I. Characteristics of patients and summary of results.

\begin{tabular}{|c|c|c|c|}
\hline & $150 \mathrm{mg}$ & $300 \mathrm{mg}$ & All arms combined \\
\hline N, Baseline (Day 28) & $12(11)$ & $14(12)$ & $26(23)$ \\
\hline Male N, Baseline (Day 28) & $10(9)$ & $11(10)$ & $21(19)$ \\
\hline Age, median (range), years & $38 \pm 10(27-65)$ & $39 \pm 10(23-65)$ & $38 \pm 10(23-65)$ \\
\hline \multicolumn{4}{|l|}{ Viral load (copies/ml) } \\
\hline Baseline & $21964 \pm 19432$ & $24383 \pm 20237$ & $23173 \pm 19400$ \\
\hline Day 28 & $17715 \pm 10803$ & $21037 \pm 13085$ & $19448 \pm 11897$ \\
\hline Follow-up (Day 56) & $38297 \pm 69411$ & $17604 \pm 13356$ & $26472 \pm 46210$ \\
\hline Day 28 vs. baseline (p) & n.s. & n.s. & n.s. \\
\hline \multicolumn{4}{|l|}{$\mathrm{CD}^{+}($cells/mmc) } \\
\hline Baseline & $627 \pm 343$ & $607 \pm 450$ & $616 \pm 394$ \\
\hline Day 28 & $581 \pm 304$ & $535 \pm 315$ & $558 \pm 303$ \\
\hline Follow-up (Day 56) & $568 \pm 250$ & $478 \pm 304$ & $517 \pm 280$ \\
\hline Day 28 vs. baseline (p) & n.s. & n.s. & n.s. \\
\hline \multicolumn{4}{|l|}{$\mathrm{CD}^{+}($cells $/ \mathrm{mmc})$} \\
\hline Baseline & $1304 \pm 505$ & $1180 \pm 610$ & $1239 \pm 553$ \\
\hline Day 28 & $1063 \pm 484$ & $984 \pm 430$ & $1024 \pm 449$ \\
\hline Follow-up (Day 56) & $1077 \pm 484$ & $883 \pm 511$ & $966 \pm 537$ \\
\hline Day 28 vs. baseline (p) & n.s. & n.s. & n.s. \\
\hline \multicolumn{4}{|l|}{ Cortisol (m.U.I./ml) } \\
\hline Baseline & $465 \pm 92$ & $380 \pm 132$ & $404 \pm 126$ \\
\hline Day 28 & $697 \pm 182$ & $977 \pm 265$ & $830 \pm 262$ \\
\hline Follow-up (Day 56) & $321 \pm 96$ & $355 \pm 155$ & $341 \pm 131$ \\
\hline Day 28 vs. baseline (p) & 0.02 & $<0.001$ & $<0.001$ \\
\hline \multicolumn{4}{|l|}{ Potassium (mEq/l) } \\
\hline Baseline & $4.3 \pm 0.4$ & $4.0 \pm 0.24$ & $4.1 \pm 0.3$ \\
\hline Day 28 & $4.0 \pm 0.3$ & $3.7 \pm 0.34$ & $3.9 \pm 0.4$ \\
\hline Follow-up (Day 56) & $4.3 \pm 0.5$ & $4.0 \pm 0.34$ & $4.2 \pm 0.4$ \\
\hline Day 28 vs. baseline (p) & n.s. & 0.02 & n.s. \\
\hline
\end{tabular}

n.s., not significant.

levels of transaminases that were not related to the treatment as they were present at baseline.

In the $300 \mathrm{mg}$ dose group, two out of 14 patients dropped out of the study on Days 7 and 9 because of severe hypokalemia and gastrointestinal discomfort and due to the occurrence of multi-metameric herpes zoster, respectively. An additional patient was put in temporary suspension on Day 21 because of gastrointestinal discomfort. He commenced treatment the day after.

In spite of this overall good clinical tolerability of the drug, hematochemical abnormalities were observed throughout the study period and were primarily related to the development of hypokalemia that occurred during at least one visit in 1 of 12 patients $(8.3 \%)$ in the $150 \mathrm{mg}$ dose group and in 7 of 14 patients $(50 \%)$ in the $300 \mathrm{mg}$ dose group.

A substantial and significant increase in the blood levels of cortisol was observed in 5 of $12(41.7 \%)$ patients of the $150 \mathrm{arm}$ group. Its maximal mean value was 1.6-fold that of baseline and it declined to normal values in all the patients on Day 56 (Fig. 1, Table I).

In the group of patients receiving $300 \mathrm{mg} /$ day mifepristone, increased blood levels of cortisol were found in $100 \%$ of the patients. Its maximal mean value was 2.7-fold that of baseline, it peaked on Day 21 and it appeared to decline to normal values in most (10/14) patients upon drug interruption at follow-up, Day 56 (Fig. 1, Table I).

Furthermore, relative to baseline values, there were also other significant abnormalities in the hematochemical analyses in the $300 \mathrm{mg}$ dose group (hyperglycemia $-14.3 \%$, increase in AST/ALT blood levels, 42.3\%) observed throughout the study period and most of them reverted to normal values at the follow-up visit 4 weeks after drug interruption.

With regard to virological and immunological data, there were no significant differences throughout the study period 


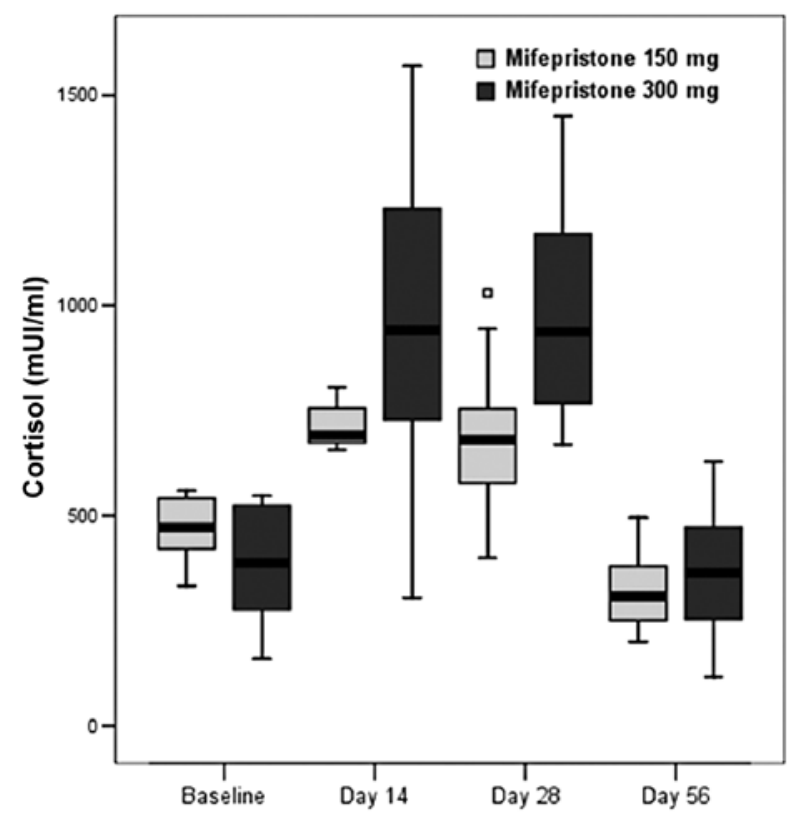

Figure 1. The effects of mifepristone treatment on blood cortisol levels in HIV-infected patients. HAART-naïve HIV-infected patients were screened -90 to -14 days prior to mifepristone administration and treated with once daily oral administration of $150 \mathrm{mg}$ (12 subjects) or $300 \mathrm{mg}$ (14 subjects) of mifepristone, starting on Day 0 for 28 consecutive days. A significan increase in the blood levels of cortisol was observed in $5(41.7 \%)$ patients treated with $150 \mathrm{mg} /$ day mifepristone and in all the patients treated with $300 \mathrm{mg} /$ day. Cortisol blood levels declined to baseline values by Day 56.

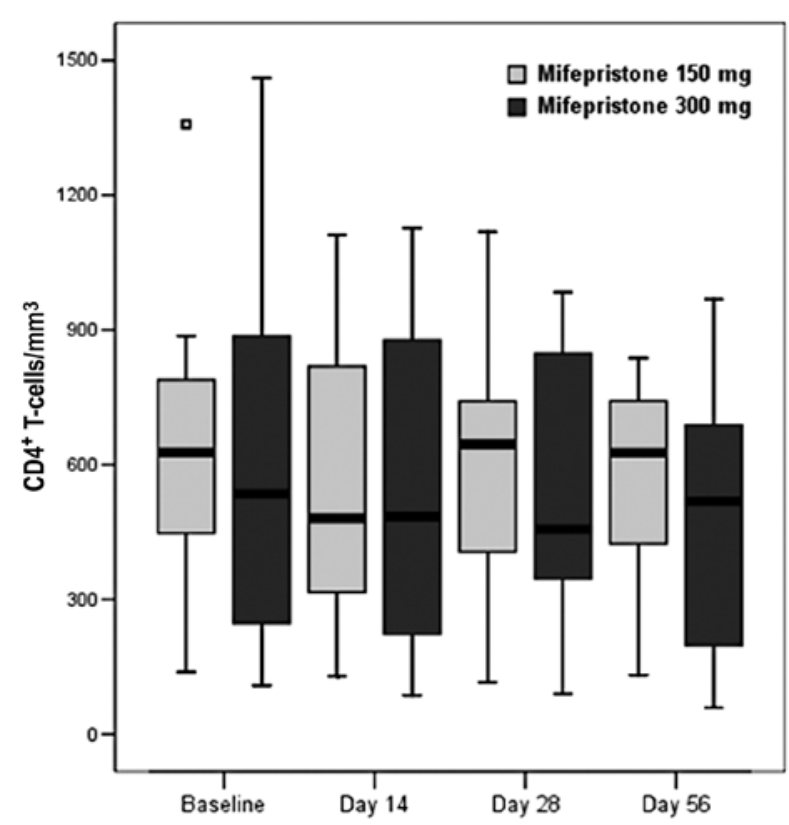

Figure 2. The effects of mifepristone treatment on $\mathrm{CD}^{+} \mathrm{T}$ cell absolute number in HIV-infected patients. HAART-naïve HIV-infected patients were screened -90 to -14 days prior to mifepristone administration and treated with once daily oral administration of $150 \mathrm{mg}$ (12 subjects) or $300 \mathrm{mg}$ (14 subjects) of mifepristone, starting on Day 0 for 28 consecutive days. Treatment with both doses failed to influence blood $\mathrm{CD}^{+} \mathrm{T}$ cell counts at all time points examined.

relative to baseline values in patients that received the $150 \mathrm{mg}$ once daily dose of mifepristone, though there was an apparent trend for an $20 \%$ reduction of the viral load on Day 28 along

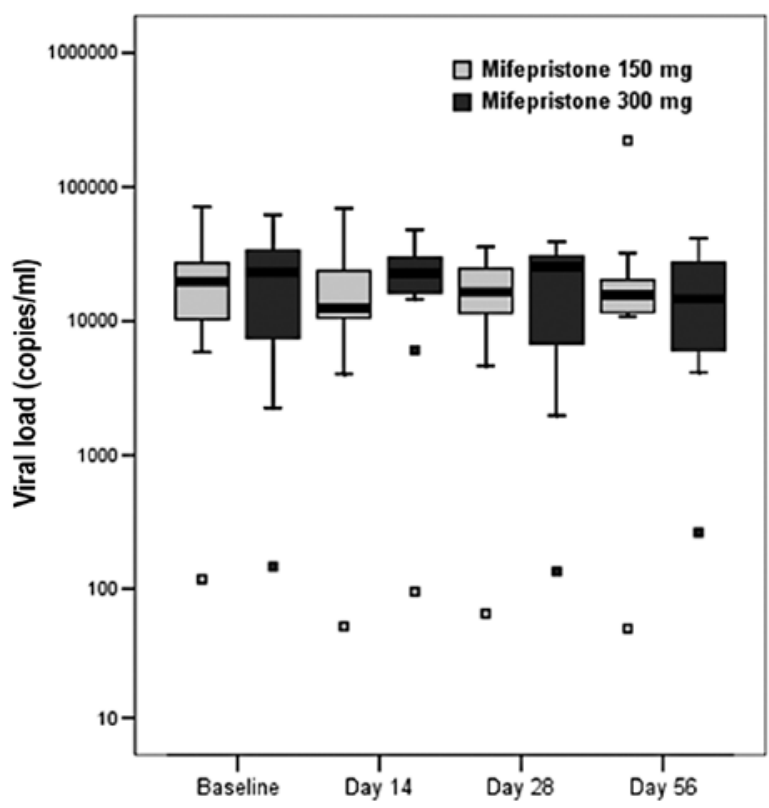

Figure 3. The effects of mifepristone treatment on HIV viral load. HAARTnaïve HIV-infected patients were screened -90 to -14 days prior to mifepristone administration and treated with once daily oral administration of $150 \mathrm{mg}$ (12 subjects) or $300 \mathrm{mg}$ (14 subjects) of mifepristone, starting on Day 0 for 28 consecutive days. Treatment with both doses failed to influence blood HIV viral load at all time points examined.

with a slight reduction of $\mathrm{CD}^{+} \mathrm{T}$ cells. None of the effects however were statistically significant (Figs. 2 and 3).

In spite of the most frequent hematochemical abnormalities observed with the highest dose of mifepristone (300 mg once daily) as compared to the $150 \mathrm{mg}$, this dose of the drug was also unable of significantly modifying either the primary or the secondary endpoints examined. There was a non-statistically significant trend towards decrease of the viral load (Table I, Fig. 3) together with a slight reduction of both $\mathrm{CD}^{+}$and $\mathrm{CD} 8^{+}$ $\mathrm{T}$ cells, the maximal peak of each of them reached at the follow-up day 56 (Table I, Fig. 2).

\section{Discussion}

Mifepristone binds to the progesterone receptor five times more avidly than progesterone. It also binds to the GR receptor four times more strongly than dexamethasone. By contrast, mifepristone binds to the androgen receptor with only one quarter of the affinity of testosterone and has essentially no binding affinity for the mineralocorticoid or estradiol receptors (24).

Although mifepristone has been assessed in a number of medical applications other than in pharmacological termination of pregnancy including the treatment of uterine fibroids, endometriosis, psychotic major depression, glaucoma, tumors and Cushing's syndrome, it has not been approved by the FDA for any of these uses. However, even the long-term use of mifepristone as an antiprogestin in myoma, meningioma and other progesterone-dependent diseases was generally welltolerated (24).

Our present data indicate that mifepristone when given as $150 \mathrm{mg}$ once daily dose was well tolerated both from the clinical and the hematochemical point of view with the exception 
of a substantial and homogeneous increase in the blood levels of cortisol that occurred in 5 of the 11 patients studied throughout the study period. The increase reverted at follow-up after discontinuation of the treatment and was not associated to clinical signs of hypercortisolism, such as hyperglycemia, hypertension or alteration of $\mathrm{Na}^{+}$and $\mathrm{K}^{+}$homeostasis.

On the other hand, the $300 \mathrm{mg}$ dose of mifepristone given for 28 consecutive days was overall less well tolerated than the lower dose. This is consistent with the higher drop out rate as well as with the higher rate of hematochemical abnormalities observed in this study. This was particularly evident with regards to the occurrence of hypokalemia that developed in 7 of 14 patients as well as to the marked increase of cortisol blood levels that were observed in all of them.

Previous studies have already shown that mifepristone may affect either the central (through its negative feedback on CRH/ACTH secretion) or peripheral actions of cortisol. Therefore, mifepristone antagonizes the negative feedback of cortisol by blocking central GRs and leading to an increase of plasma ACTH and cortisol levels that is particularly evident in the early morning (26-29).

It has also been reported that, occasionally, long-term administration of mifepristone may be associated with low serum potassium levels, a slight increase in serum creatinine levels or with a moderate elevation of hepatic enzymes $(30,31)$.

Since the GRs are blocked by mifepristone, while the mineralocorticoid receptors are not, the high cortisol levels associated with mifepristone-treatment may cause overstimulation of the latter. In light of our results, careful monitoring of potassium levels, as well as blood pressure values, during and eventually after mifepristone treatment are highly recommended. In the rare cases with clinically relevant anti-glucocorticoid side effects, concomitant glucocorticoid therapy may eventually be initiated (24).

Although neither primary nor secondary endpoints were achieved with either dose, it is worthy to mention that in the $150 \mathrm{mg} /$ day group there was a non-significant trend towards reduction of the HIV-1 plasma viral load along with diminished $\mathrm{CD}^{+} \mathrm{T}$-cell number, that was more pronounced at the end of the drug treatment on Day 28. Moreover, a similar trend as regard to the $\mathrm{CD}^{+} \mathrm{T}$-cell count was observed in the $300 \mathrm{mg} /$ day group, but it occurred with different kinetics.

In spite of a higher rate of hematochemical alterations and patient drop-out, the highest dose of mifepristone failed to significantly modify viro-immunological parameters, such as the viral load or the $\mathrm{CD}^{+}$and $\mathrm{CD}^{+} \mathrm{T}$-cell counts in these patients. The trend toward a reduced viral load and $\mathrm{CD}^{+} \mathrm{T}$ cells was comparable to that observed in the $150 \mathrm{mg} /$ day dose group, but it occurred with different kinetics; it peaked in the follow-up period on Day 56, e.g. 4 weeks after interruption of the drug. A slight reduction of $\mathrm{CD}^{+} \mathrm{T}$ cells was also observed and it reached its maximal value at the follow-up period on Day 56.

These data prove that when given at the doses examined in the present study, mifepristone is not able to significantly influence viro-immunological parameters in $\mathrm{HIV}$-infected patients.

The discordance of the results of the in vitro system and macaque model and those in our present trial may depend on pharmacokinetic differences between the two experimental settings that have been discussed elsewhere (25) as well as on possible differences in the pathogenesis of SIV infection in macaques and HIV infection of humans. In a more general context, these data reinforce the need of exerting caution when preclinical data generated with SIV in macaque are translated to the clinical setting.

The lack of antiviral activity of mifepristone in our study is consistent with a previous phase I/II trial that showed that administration of mifepristone at 75-225 mg daily doses was safe and well-tolerated, but it did not significantly influence plasma HIV-1 RNA levels and CD4+ lymphocyte count compared to the baseline (25).

The trend to reduced HIV viral load observed with the $150 \mathrm{mg}$ dose of the drug along with the strong preclinical data and coupled to the good clinical tolerability would justify additional studies of the additive or synergistic effects of mifepristone with other anti-HIV drugs if there were not so many alternative options for the treatment of HIV-infected patients that also successfully cover, and with increasingly limited side-effects, those HIV patients that develop resistance to one therapeutic strategy. Nonetheless, the slight (if any) anti-retroviral activity of mifepristone coupled with its pharmacological profile may allow the postulation of an alternative use for mifepristone in the HIV setting that relates to the prevention and treatment of HIV-associated Kaposi sarcoma (KS). In fact, it has recently been shown that cultured KS cells from AIDS patients express an unusually high level of GR protein, that is further up-regulated by glucocorticoid treatment. In addition, in vitro treatment with a potent agonist of the GRs, such as dexamethasone significantly stimulated the proliferation and survival of sarcoma cells and these stimulatory effects were completely abolished by treatment with mifepristone (32).

The development of novel therapeutic options in the treatment of KS remains an important medical need even during the HAART era. In fact, KS remains the second most frequent tumor in HIV-infected patients worldwide, and it has become the most common cancer in Sub-Saharan Africa. In western countries the risk for KS in homosexual men is 5-10 times higher compared to other groups of individuals practicing other HIV-risk behaviors. Patients with KS in Sub-Saharan Africa have very high tumor burdens and rapid disease progression with a diminished life expectancy of less than 6 months. Several different therapeutic options are available, but the optimal therapy is still unclear. HAART including protease inhibitors may represent the first treatment step for slowly progressive disease; chemotherapy plus HAART is indicated for visceral and/or rapidly progressive disease, whereas maintenance HAART after systemic chemotherapy may be an effective anti-KS measure after debulking chemotherapy (33).

Taken together and in light of our present findings we believe that preclinical studies may be warranted to assess the hypothesis that treatment with mifepristone may represent a novel therapeutic strategy for the treatment of some cases of HIV-related KS.

\section{References}

1. Connor RI, Chen BK, Choe S and Landau NR: Vpr is required for efficient replication of human immunodeficiency virus type 1 in mononuclear phagocytes. Virology 206: 935-944, 1995. 
2. Hattori N, Michaels F, Fargnoli K, Marcon L, Gallo RC and Franchini G: The human immunodeficiency virus type $2 \mathrm{vpr}$ gene is essential for productive infection of human macrophages. Proc Natl Acad Sci USA 87: 8080-8084, 1990.

3. Sherman MP, de Noronha CM, Eckstein LA, et al: Nuclear export of $\mathrm{Vpr}$ is required for efficient replication of human immunodeficiency virus type 1 in tissue macrophages. J Virol 77 7582-7589, 2003.

4. Schafer EA, Venkatachari NJ and Ayyavoo V: Antiviral effects of mifepristone on human immunodeficiency virus type-1 (HIV-1): targeting Vpr and its cellular partner, the glucocorticoid receptor (GR). Antiviral Res 72: 224-232, 2006.

5. Thieu KP, Morrow MP, Shedlock DJ, et al: HIV-1 Vpr: regulator of viral survival (Review). Curr HIV Res 7: 153-162, 2009.

6. Goh WC, Rogel ME, Kinsey CM, et al: HIV-1 Vpr increases viral expression by manipulation of the cell cycle: a mechanism for selection of Vpr in vivo. Nat Med 4: 65-71, 1998.

7. Ayyavoo V, Mahboubi A, Mahalingam S, et al: HIV-1 Vpr suppresses immune activation and apoptosis through regulation of nuclear factor $\kappa$ B. Nat Med 3: 1117-1123, 1997.

8. Subbramanian RA, Kessous-Elbaz A, Lodge R, et al: Human immunodeficiency virus type $1 \mathrm{Vpr}$ is a positive regulator of viral transcription and infectivity in primary human macrophages. J Exp Med 187: 1103-1111, 1998.

9. Cohen EA, Terwilliger EF, Jalinoos Y, Proulx J, Sodroski JG and Haseltine WA: Identification of HIV-1 vpr product and function. J Acquir Immune Defic Syndr 3: 11-18, 1990.

10. Kino T, Gragerov A, Kopp JB, Stauber RH, Pavlakis GN and Chrousos GP: The HIV-1 virion-associated protein vpr is a coactivator of the human glucocorticoid receptor. J Exp Med 189: 51-62, 1999.

11. Sawaya BE, Khalili K, Gordon J, Taube R and Amini S: Cooperative interaction between HIV-1 regulatory proteins Tat and $\mathrm{Vpr}$ modulates transcription of the viral genome. J Biol Chem 275: 35209-35214, 2000

12. Sherman MP, de Noronha CM, Pearce D and Greene WC: Human immunodeficiency virus type $1 \mathrm{Vpr}$ contains two leucine-rich helices that mediate glucocorticoid receptor coactivation independently of its effects on G2 cell cycle arrest. J Virol 74 8159-8165, 2000.

13. Paxton W, Connor RI and Landau NR: Incorporation of Vpr into human immunodeficiency virus type 1 virions: requirement for the p6 region of gag and mutational analysis. J Virol 67: 7229-7237, 1993.

14. Popov S, Rexach M, Zybarth G, et al: Viral protein R regulates nuclear import of the HIV-1 pre-integration complex. EMBO J 17: 909-917, 1998

15. Heinzinger NK, Bukinsky MI, Haggerty SA, et al: The Vpr protein of human immunodeficiency virus type 1 influences nuclear localization of viral nucleic acids in nondividing host cells. Proc Natl Acad Sci USA 91: 7311-7315, 1994.

16. Vodicka MA, Koepp DM, Silver PA and Emerman M: HIV-1 $\mathrm{Vpr}$ interacts with the nuclear transport pathway to promote macrophage infection. Genes Dev 12: 175-185, 1998.

17. Mirani M,Elenkov I, Volpi S, Hiroi N, Chrousos GP and Kino T: HIV-1 protein Vpr suppresses IL-12 production from human monocytes by enhancing glucocorticoid action: potential implications of $\mathrm{Vpr}$ coactivator activity for the innate and cellula immunity deficits observed in HIV-1 infection. J Immunol 169: 6361-6368, 2002.

18. Levy DN, Refaeli Y, MacGregor RR and Weiner DB: Serum Vpr regulates productive infection and latency of human immunodeficiency virus type 1.Proc Natl Acad Sci USA 91: 10873-10877, 1994.

19. Muthumani K, Choo AY, Premkumar A et al: Human immunodeficiency virus type 1 (HIV-1) Vpr-regulated cell death: insights into mechanism. Cell Death Differ 1 (Suppl 1): S962S970, 2005.

20. Lamberts SW, Koper JW and de Jong FH: The endocrine effects of long-term treatment with mifepristone (RU 486). J Clin Endocrinol Metab 73: 187-191, 1991.
21. Lamberts SW, Tanghe HL, Avezaat CJ, et al: Mifepristone (RU 486) treatment of meningiomas. J Neurol Neurosurg Psychiatry 55: 486-490, 1992.

22. Grunberg SM, Weiss MH, Spitz IM, et al: Treatment of unresectable meningiomas with the antiprogesterone agent mifepristone. J Neurosurg 74: 861-866, 1991.

23. Rocereto TF, Saul HM, Aikins JA Jr and Paulson J: Phase II study of mifepristone (RU486) in refractory ovarian cancer. Gynecol Oncol 77: 429-423, 2000

24. Johanssen S and Allolio B: Mifepristone (RU 486) in Cushing's syndrome. Eur J Endocrinol 157: 561-569, 2007.

25. Para MF, Schouten J, Rosenkranz SL, et al: Phase I/II trial of the anti-HIV activity of mifepristone in HIV-infected subjects ACTG 5200. J Acquir Immune Defic Syndr 53: 491-495, 2010.

26. Bertagna X, Bertagna C, Luton JP, Husson JM and Girard F: The new steroid analog RU 486 inhibits glucocorticoid action in man. J Clin Endocrinol Metab 59: 25-28, 1984.

27. Bertagna X, Escourolle H, Pinquier JL, et al: Administration of RU 486 for 8 days in normal volunteers: antiglucocorticoid effect with no evidence of peripheral cortisol deprivation. J Clin Endocrinol Metab 78: 375-380, 1994.

28. Healy DL, Chrousos GP, Schulte HM, et al: Pituitary and adrenal responses to the antiprogesterone and anti-glucocorticoid steroid RU 486 in primates. J Clin Endocrinol Metab 57: 863-865, 1983.

29. Gaillard RC, Riondel A, Muller AF, Herrmann W and Baulieu EE: RU 486: a steroid with antiglucocorticosteroid activity that only disinhibits the human pituitary-adrenal system at a specific time of day. Proc Natl Acad Sci USA 81: 3879-3882, 1984.

30. Spitz IM: Progesterone antagonists and progesterone receptor modulators: an overview. Steroids 68: 981-993, 2003.

31. Eisinger SH, Bonfiglio T, Fiscella K, Meldrum S and Guzick DS: Twelve-month safety and efficacy of low-dose mifepristone for uterine myomas. J Minim Invasive Gynecol 12: 227-233, 2005.

32. Guo WX and Antakly T: AIDS-related Kaposi's sarcoma: evidence for direct stimulatory effect of glucocorticoid on cell proliferation. Am J Pathol 146: 727-734, 1995.

33. Martellotta F, Berretta M, Vaccher E, Schioppa O, Zanet E and Tirelli U: AIDS-related Kaposi's sarcoma: state of the art and therapeutic strategies. Curr HIV Res 7: 634-638, 2009.

\section{Note added in proof}

At the stage of proofreading a paper has been published describing an AlphaScreen ${ }^{\circledR}$-based assay for high-throughput screening for specific inhibitors of nuclear import of HIV through the integrase molecule of HIV-1 (1). By screening for specific inhibitors of the interaction between integrase and its nuclear transport receptor importin $\alpha / \beta$, the authors have identified mifepristone as one of the inhibitors of the integrase/ importin $\alpha / \beta$ interaction. Mifepristone was effective in preventing active nuclear transport of integrase in transfected cells. This study sheds new lights on the in vitro anti-HIV-1 activity of mifepristone. Additional studies with this drug in HIV-1 infected patients are required in order to understand and possibly overcome the factors limiting its activity in vivo as well as to evaluate its efficacy in the context of HAART regimes.

1. Wagstaff KM, Rawlinson SM, Hearps AC and Jans DA: An AlphaScreen ${ }^{\circledR}$-based assay for high-throughput screening for specific inhibitors of nuclear import. J Biomol Screen 16: 192-200, 2011. 